\title{
Towards the Integration of Technological, Organizational and Human Subsystems of Organizations to Enhance Productivity
}

\author{
M. A. Sanda ${ }^{1}$ J. Johansson ${ }^{1}$ \\ ${ }^{1}$ Centre of Advanced Mining and Metallurgy (CAMM), Division of Human Work Sciences, Department of Business \\ Administration, Technology and Social Sciences, Luleå University of Technology, Sweden. \\ Email: mohami@1tu.se, jan.johansson@1tu.se
}

\begin{abstract}
This paper looks at the challenges that most organizations, especially in the mining industry, face in managing both technological and human activities along their value chains. The aim was to understand how the organization's technological, organizational and human components could be integrated to enhance collaboration and productive efficiency. Guided by an organizational activity theory framework, qualitative data were collected through interviews in a mining firm. The analysis of contradictions showed that production work in the mine is challenged due to the lack of proper coordination in task executions, with workers constrained in their task undertakings and unable to reduce downtime. It was concluded that firms can use the framework as an effective instrument, not only for organizational activity and work environment analyses, but as a tool for learning how to understand and manage the systemic characteristics of the technological, organizational and human components of their organizational activity systems.
\end{abstract}

Keywords - Productivity, organizational activity, human subsystem, technological subsystem, organizational subsystem.

\section{INTRODUCTION}

The mining industry faces a number of challenges which must be addressed with a socio-technical approach that cover the whole mining and minerals chain including environmental issues. Mining industry need a new mental image of oneself based on new technology and a modern work organization that supports both high productivity and good working conditions. There is a need for a new holistic vision for the mine of the future [1]. The longerterm challenges include environmental issues, but any future mining must not endanger the health and welfare of local inhabitants and post-mining there should be as few traces as possible in nature. Another challenge is the problem of recruiting skilled workers to the mines often located far from larger communities. A modern mine is so technically advanced that the proportion of unskilled labor will decrease significantly or disappear. There will be fewer workers with higher wage costs. Most mining companies want to avoid a "fly-in" and "fly-out" situation, as this could create social instability of societies and cause trouble in the workplace. This requires an organization of the work that supports both high productivity and good working conditions. A further challenge is to break the unequal gender balance that exists in most mines. It is not just about recruiting more women, but rather to challenge the prevailing macho culture in order to create a safer and more productive environment [1].

According to [2], the current skills shortage will compel the industry to increase productivity per worker, and key enablers will be automation and remote operation technologies. This includes smart mining systems to automate business processes and operational decisions, supported by advanced analytics, collaborative visualization systems, and improved application of remote and automation technologies to plant and equipment. Recognizing the need to improve collaboration across supply chains will lead to a focus on improved business integration, including more mature partnering models. Increasingly, mining enterprises will seek to collaborate closely with partner organizations, creating 'virtual' and 'extended' enterprises. The mining companies will evolve appropriate technologies, business processes and organizational structures to allow them to operate as extended virtual enterprises [2]. These enterprises will seek to link in collaboration partners, such as service contractors, support and supply organizations, as well as expert services. Remote operations centers will function as 'collaboration hubs', facilitating integration of business process across the supply chain [2]. This paper looks at this challenge at the workplace with the aim of creating knowledge on the best way to integrate "Technological, Organizational and Human Systems" in the development of intelligent automation process. This is necessitated by the observation that, in recent times, concern over the gap between the theory of what people do and what people actually do has given rise to the 'practice' approach in management literature focusing upon the way that actors interact with the social and physical features of context in the everyday activities that constitute practice [3].

The need for this research is derived from the challenges posed by the prevailing thinking among industrial firms to the effect that by replacing humans with machines at all levels in the value chain, a rapid increase of automation and integration of various processes and unit operations can occur. By implication, the critical issue of effective operation and maintenance of production systems could be addressed in order to enhance the firm becoming economic viable and competitive. Since a production system consists of technical components, information, materials and humans, there is the need for holistic perspectives to be included in the operation, 
maintenance, engineering and control of components of the system. This therefore, requires the creation of a harmony between the technical and the social system, and whose creation can be facilitated by a holistic view of the system's functions. The significance of such harmony creation is defined by the realization that in order to enhance the development of intelligent automation systems for industrial firms, there is a need for the creation of knowledge on the harmonious integration of technological, organizational and human systems. This is because such integration will stand to provide the basis for the evolution of a community of practice at the workplace.

\section{LITERATURE REVIEW}

Organization behaviour, from the perspectives of [4], is directed toward objectives that are more or less understood by members of the group. In this context, Kast and Rosenzweig [4] view an organization from two perspectives. On the one hand, an organization uses knowledge and techniques in the accomplishment of its tasks. On the other hand, an organization implies structuring and integrating activities (i.e., people working or cooperating together in interdependent relationships). Also, since the notion of interrelatedness suggests a social system, organizations consist of psychosocial systems (with people interacting in groups), technological systems (with people using knowledge and techniques), and an integration of structured activities, (with people working together in patterned relationships) [4]. Thus by taking activity theory as it stands today into context, the bridge principle underlying it is derived from the perspectives of psychological development. In this research, the need is seen for the contextual orientation of this bridge principle to reflect the organizational perspectives whilst retaining the internal principle. In this regard, the description of an organization as outlined by [4] is found to be of relevance and thus applicable in relation to the bridge principle undying the activity theory framework.

The value of any theory, as [5] has noted, is not whether it provides an objective representation of reality, but rather how well it can shape an object of study, highlighting relevant issues. The implication derived from the research purpose is that, in order to be able to develop a functional intelligent automation system, there is the need for the development of knowledge to facilitate the integration of technological, organizational and human subsystems. One important criterion that can facilitate the attainment of such better systems would therefore be the derivation of knowledge about the positive integration of man and machine (i.e. to be able to see the human as an integrated part of the system). This is by virtue of the fact that a production system consists of both humans/people and technology. As it is noted by [6], when employees are not actively involved throughout the planning and implementation processes, the result is often a poorly designed work system and a lack of employee commitment. In the task interpretation process, the worker has to be able to involve his personal prerequisites such as experience, skills and physical constitution, as well as his/her context as part of social systems inside and outside the organization [6]. According to [6], the worker has to, additionally solve all the problems that were not taken care of, or were misinterpreted, when management designed the task. As such, employees even frequently display overt or passive-aggressive resistance to the changes. Therefore, in the process of developing new work practices in an organizational system, the key actors in the exercise also double up as learners of their new activities. As it is noted by [6], when employees are not actively involved throughout the planning and implementation processes, the result is often a poorly designed work system and a lack of employee commitment. In the task interpretation process, the worker has to be able to involve his personal prerequisites such as experience, skills and physical constitution, as well as his/her context as part of social systems inside and outside the organization [6]. According to [6], the worker has to, additionally solve all the problems that were not taken care of, or were misinterpreted, when management designed the task. As such, employees even frequently display overt or passive-aggressive resistance to the changes.

The framework for exploring an organization's activity system (figure 1 below) adapted from [7] is used to appraise the quality of the organizational, technological and human systems' relationships.

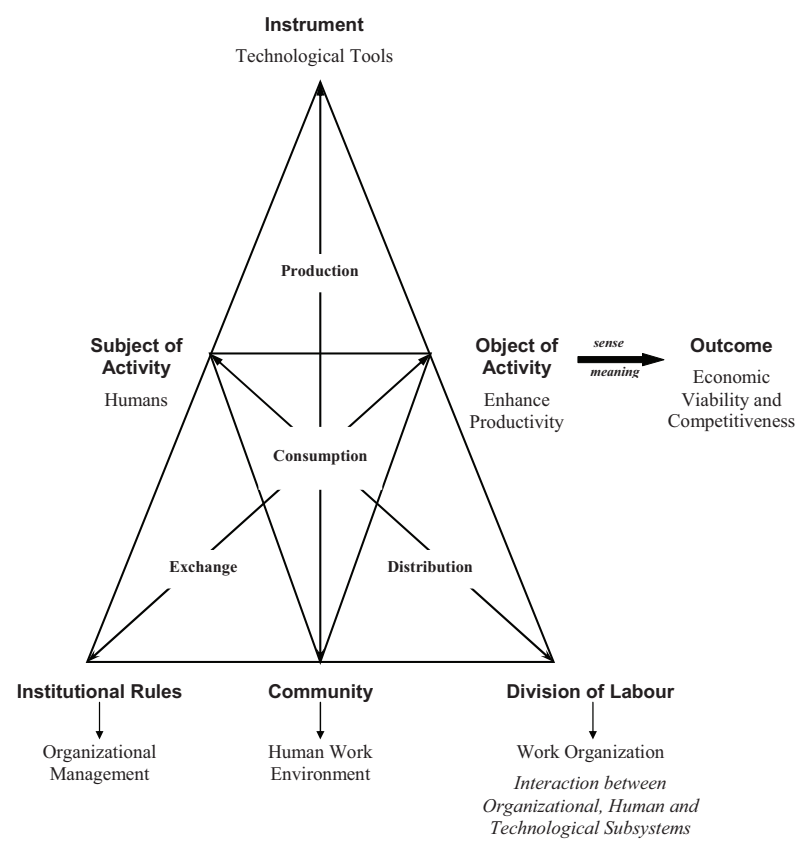

Fig. 1. Framework for learning and dealing with the systemic character of an organization's activity system [adapted from 7]

The framework (figure 1 above) allows for the understanding of specific mediational elements in the institutional rules that impact on organizational 
management issues, such as the organizational structure, corporate culture. It also allows for the measurement of specific mediational elements related to the organization's community which impact on the human work environment, such as discourse, trust, social interaction, level of commitment, and degree of competence.

The framework also allows for the understanding of the effectiveness and reliability of the mediational elements that defines the way labour is divided and work organized among humans and technological tools. In this context, specific elements such as the human resource base, the medium of discourse, trust, appropriation, cooperation, collaboration, networking, and social relationship can also be measured.

As it is explained by [7], analyzing organizations as activity systems encourages people to stand back from their everyday routines and to perceive the overall pattern that such routines fall into. It encourages exploration of the origins of these patterns, of what presently sustains them, of the imagery used in descriptions of them, of the inconsistencies and conflicts that they embrace, and of the stimulation that new challenges may provide.

\section{METHODOLOGY}

Data were collected in a mining firm in Ghana using the interview approach, as well as the techniques of observation and video recordings of production work in the mines. The interviewees were comprised of 8 managers responsible for the management of the mine's organizational, technological and human resources systems. They included the mine's production manager, two mine captains, the human resource manager, the environment manager, the technical systems manager, the business re-engineering manager, and the chief rock engineer/geologist.

Analysis of contradictions ([8] [7]) was used to identify the key systemic components of the organization's activity systems for which possible conflicts and troubles can emerge in the technological, organizational and human systems integration processes. Using the template shown in figure 1 above as a guide, further analysis was conducted to identify systemic components of the mine's organizational activity system in order to understand as well as find ways on how to manage specific conflicts and troubles that can possibly emerge to constraint the functionality of the technological, organizational and human system integration process.

\section{RESULTS AND DISCUSSION}

In order to enable the understanding of the functionalities of the mine's organizational, technological, and human subsystems, the relational effect of both individual and collective activities embedded in the different components of the mine's organizational activity system are qualitatively appraised and discussed.
It is learnt from the interviews that the work activities in the mine is underlined by a business practice model basically known as the Business Process Forum (BPF). The premise for using this model is that it provides a better chance of achieving the mine's production target by enhancing job planning, scheduling and resourcing ahead of time. Application of the model to guide production activities also allows for the attainment of cost reduction and performance improvement. The manager responsible for implementing the BPF model observed in the interview that all the firm's stakeholders, including the superintendents and supervisors were engaged and also coached to understand the functional principles of the BPF model. In order to underscore this observation, the interviewees who are managers provided the following overviews of how all stakeholders engaged in the production activity are engaged;

We have a group of workers called role holders who play various roles in the mining activities. Each group is comprised of a planner, a resource coordinator and a scheduler. These guys sit together were the miners are working. If a miner sees any work which he thinks is important to be done, he goes to these role holders. They in turn sit with the concerned miner(s) to plan the job, schedule it, look at the resources we have, and then get it executed. This way of doing things brought everybody in the picture.

The observation above showed that newer technologies have brought to the fore other business models into the frame of mining production activity. Such models, as it is the case with the firm's BPF model, enhance the monitoring process of work activity on an hourly and daily basis. It also enhances the planning process of work activity on a monthly and yearly basis. This trend will move further to the real-time mining processes that involve minute by minute operational control decisions [8]. Such trend move has a direct consequence on the firm's organizational management; organizational structures become flatter, and a comparatively smaller workforce with less supervisory roles and a greater emphasis on collaborating with partner organizations emerges. Automation and remote operations centers will also enable a reduction in the on-site workforce. As it is explained by the interviewees;

There is also the management aspect were work has to be done during the month. The role holders will take them, sit with the superintendents or mine captains and plan these jobs. The planning in this instance mean that breaking the jobs into various activities and each one of them is scheduled. They identify the resources that will be needed and once that has been agreed with a mine captain, it becomes his baby to follow. There is a system of monitoring to ensure that you achieve your target.

A smaller workforce with expanded role responsibilities creates the risk of losing operational and 
business knowledge. Emerging technologies that intelligently capture and transfer knowledge and increase situational understanding will be adopted by progressive organizations to mitigate the risk [8]. In this regard, the major dimension differentiating levels of maturity in the supply chain industry was the level of collaboration across the value chain and involving more and more external entities in the network. As the enterprise of the future becomes increasingly 'extended' and 'virtual', business processes will more easily span organizational boundaries, and companies will need to adopt strategies to allow evolving business models to keep pace with more effective collaboration [8]. In the view of [8], the future working environment will consist of flat organizational structures, driven by necessity and enabled by technology. This will mean a leaner, data and technology savvy workforce with cross-skilled employees, with most supervisory roles conducted from operations centers located conveniently in major urban centers. As a result, technology assisted workers will perform the job of two or three traditional roles with an emphasis on monitoring, problem solving and operational improvement [8]. As it is explained by an interviewee;

Managers are encouraged to take ownership of the system. The role holder's are at the same level as the mine captains. They have the power to talk to the mine captains; otherwise, the captains will trample over them. However, the role holders do not work for the mine captains, but rather for the managers. They advise the managers. So that is how we have done it and everybody is in the system. That has taken away ownership from the system.

The assessment of the relational impact of different components of production activities in the mine by the interviewees who are mine captains showed that the planning of production work underground is a key challenge. Production work underground is described by the interviewees as entailing lots of challenges due to the lack of proper coordination in task executions.

Sometimes when you look at what happens it looks like we are sitting in different saloons and everybody is looking at he can do within the mine. Even though we share office boundaries, we are not talking to each other. So, it is like the job that needs to be executed, you need inputs from other disciplines which has to be done and you also have to get yours done before you can do it.

As a consequence, workers who are directly engaged with specific tasks are constrained in their task undertakings and are thus, unable to reduce downtime. The underlying view for this observation is underscored by the interviews are it is highlighted in the following excepts;

Yes, we are all trying to make production activity better because our lives depend on it. The managers have a very fine model. Why? They sit by the computer easily came out with such model. We sit over here and tell them that this is our activity. This is the resource that we need. When we try to get some of this resource from the stores, we encounter a lot of problems. At times, by the time it comes, there is whole lot of lag. So, you see a lot of frustration and irritation coming in, and affecting the people who are supposed to do the work. And the whole stores supply coming late imply that a whole lot of downtime has come. So, all this adds to the frustration of the mine captains.

The derivation from the interviewees' observations above is that skills and knowledge should not be seen as things that are simply static and accumulated by individuals, but rather as things that are created and changed in socio-cultural contexts, through individual as well as collective processes [1]. The implication being that knowledge, as it is derived from the interview excerpt below, is not only a question of individual behaviour, but also about organization, power and identity [9].

We do not come together to sit and discuss issues so that everybody knows who is supposed to do what and when to start and finish so I can come in as production manager. This communication gap is what is creating all these problems resulting in unnecessary waste of time, because people think that this man must finish his before I come in, but that man sitting there might not even know that your work depends on him. So I see it as a problem If we are not talking to one another and finding solutions to the work.

The transformation of knowledge, whether it is a question of upskilling, deskilling or re-skilling, has effects on workplace culture and identities [1]. This means that the changes in qualifications, together with the new technology, the better work environment, and the new type of work tasks, challenge old behaviours and attitudes. This process is neither simple and nor without resistance [1]. This is because, increasing levels of collaboration and integration with partner organizations, as well as trends towards the 'service oriented enterprise', will see job roles shift to external service providers [8]. This will involve more collaboration with external experts and the orchestration of services provided both within and external to the organization. The relevance of such collaboration in the firm is deemed very important as it is highlighted by the following excerpt;

The leadership of the mine must call a stakeholder meeting and bring all the parties together to discuss what activities need to be done, in relation to who is supposed to do what, when is it supposed to get done, and what resources are needed that are not available, so that the manager can influence certain decisions and get things through early enough so that work can start early enough based on whatever plans that have been put in place. That is what I see as not in place. 
As such, teams of specialists will be increasingly required to solve problems rapidly, for example, allowing logistics schedulers, mine planners and maintenance schedulers to come together to run simulations and rapidly adapt the production schedule in response to a new market opportunity. This type of rapid problem solving requires experts that can interact with each other and perform advanced analytics on data in a collaborative environment supported by appropriate technology [8].

Creating work identity involves learning [1]. For example, individuals need to figure out what identities to enact, how to enact them and how others perceive their identity. Individuals' actions and identity formation at work will tend to be affected by the organizational structures and/or the symbols characteristic of the production system in different ways. This can be understood as both socialization and learning, that is, as a part of the process of becoming a full member of an organization or a community of practice ([10] [11]). In a community of practice, individual thought is essentially social and is developed in interaction with the practical activities of a community, through living and participating in its experiences over time [12]. Thus, to understand practice, it is important to move beyond institutional similarities to penetrate the situated and localized nature of practice in particular contexts. Practice must be seen as local and situated, arising from the moment-by-moment interactions between actors, on one hand, and between actors and the environments of their action, on the other hand [12]. In the case of the organizations, the issue here is not only about designing systems/automation that is adaptable to humans. It also calls for the humans to be regarded as unique resources when designing better systems. this is because, people's sense of their own knowledge in work, and the skills valued by the group to which they see themselves belonging, forms a critical element of their identity [9]. Together with the more formal structural aspects at the workplace (i.e. technology, organizational structure and qualification demands), the identity aspects of work, the symbolic aspects of work, that is, stories, myths, ideas and perceptions of what a 'real' worker is like, and relations form four organizational processes [9]. In the mining industry, working life, work organization, workplace culture and learning are seen as important factors to a company's ability to develop an effective, flexible and yet sustainable production system. There is also a rising awareness that gender is one aspect of this, even if the companies experience difficulties in their practical measures.

\section{CONCLUSION}

This study delved on the need for learning to be enabled on how to effectively integrate and manage the functionality of the technological, organizational and human subsystems of organizations. A broaden perspectives and new angles on current production problems which will lead to the development of the requisite organizational activity system towards meeting the mining industry's need for efficient and effective productivity was appraised. It is concluded firms can use the framework as an effective instrument, not only for organizational activity and work environment analyses, but as a tool for learning how to understand and manage the systemic characters of their organizational activity systems. This study is viewed as timely and of relevance to both academia and industry as it stands to provide the needed knowledge on how organizations can enhance work efficiency, productivity and work environments.

\section{REFERENCES}

[1] L. Abrahamsson and J. Johansson, "Future mining: Workers' skills, identity and gender when meeting changing technology", in Proc. 1st International Future Mining Conference and Exhibition, S. Sayden, Ed. Sydney, Australia: The Australian Institute of Mining and Metallurgy, 2008, pp. $213-220$.

[2] J. Bassan, V. Srinivasan, P. Knights, and C. T. Farrelly, "A Day in the Life of a mine worker in 2025", in Proc. 1st International Future Mining Conference and Exhibition, S. Sayden, Ed. Sydney, Australia: The Australian Institute of Mining and Metallurgy, 2008, pp. $71-78$.

[3] M. A. Sanda and Y. Fältholm (2005), in “Engeström's triangular model as a representation of an organization's activity system: A framework for the study of practice in organizations", in Proc. 1st ISCAR Conference. Sevilla, Spain. $20^{\text {th }}-24^{\text {th }}$ September 2005. pp. 739-741.

[4] F. E. Kast and J. E. Rosenzweig. Organization and Management: A Systems and Contingency Approach. New York: McGraw-Hill, 1985.

[5] G. Z. Bedny, and W. Karwowski, "Activity theory as a basis for the study of work", Ergonomics, vol. 47, no. 2, pp. 134-153, 2004.

[6] M. A. Sanda, "Exploring the concept of emerging object of activities in understanding the complexity of managing organizational change", in Symposium Book of Abstracts, 2nd ISCAR Conference. San Diego, Southern California, 813 September, 2008. pp. 34.

[7] M. A. Sanda. Four Case Studies on the Commercialisation of Government R\&D Agencies: An Organizational Activity Theoretical Approach. Doctoral Thesis 2006:33/ISSN:1402-1544. Luleå, Sweden: Luleå University of Technology Press, 2006.

[8] Y. Engeström, "Expansive learning at work: Toward an activity theoretical reconceptualization". Journal of Education and Work, vol. 14, no. 1, pp. 133-156, 2001.

[9] T. Fenwick, "Learning as grounding and flying: Knowledge, skill and transformation in changing work contexts. Journal of Industrial Relations, vol. 48, no. 5, pp. 691-706, 2006.

[10] M. Salminen-Karlsson. Situating Gender in Situated Learning: Experiences from a Computer Company. Stockholm: Centrum för Kvinnoforskning - Stockholms Universitet, 2003.

[11] E. Wenger. Communities of Practice. New York: Cambridge University Press, 1998.

[12] J. Lave and E. Wenger. Situated Learning: Legitimate Peripheral Participation. Cambridge, UK: Cambridge University Press, 1991. 